\title{
A Phase Modulation Scheme for Millimeter Wave Generation Based on Frequency Octupling using $\mathrm{LiNbO}_{3}$ Mach- Zehnder Modulator.
}

\author{
Anand Prem $\mathrm{P} \mathrm{K}^{\# 1}$, Arvind Chakrapani ${ }^{\# 2}$ \\ "Department of Electronics and Communication Engineering, \\ Karpagam College of Engineering, Coimbatore, Tamil Nadu, India \\ 19anandprem.2008@gmail.com \\ 2arvichakra@gmail.com
}

\begin{abstract}
A $80 \mathrm{GHz}$ millimeter wave (mmw) has been generated with a single Lithium niobate Mach Zehnder Modulator ( $\mathrm{LiNbO}_{3}$ MZM) using phase modulation (PM) scheme based on frequency octupling with a modulation voltage of $9 \mathrm{~V}$. The main advantage of this experiment is that the carrier is suppressed by changing the phase of the signal in the modulator and the need for an optical filter for suppressing the carrier signal is eliminated. Moreover the experiment shows a better data rate, less hardware complexity and less cost for its realization. The generated optical signal is modulated by the data signal in the optical domain which is transmitted through a single mode fiber for a distance of $70 \mathrm{~km}$. The received signal is detected using a photodiode to reconstruct the $\mathbf{m m w}$ and further its optical spectrum, RF spectrum and eye diagram are obtained by means of simulation to ensure the robustness of the proposed method. The method achieves error free transmission which is evident from the eye diagram and the efficiency is ensured by the quality factor plotted by the BER analyzer.
\end{abstract}

Keyword- Lithium Niobate Mach-Zehnder modulator; Radio over Fiber; Phase Modulation; Optical Spectrum; Millimeter wave generation; Frequency Octupling.

\section{INTRODUCTION}

In recent days, the optical communication demands use of mmw range signals for data transmission through an optical fiber [1], [2]. The mmw has huge bandwidth and capacity which supports transfer of data rate in the range of $100 \mathrm{Gbps}$ without any delay and congestion [3]. In future the wireless and space communication will be most common method used due to the high bandwidth provided by the mmw signal for data transfer [4], [5]. Since data rate and quality of the signal in optical domain is high when compared to other conventional methods in electrical domain [6], loss less data transmission is carried out by using light waves [7]. The electrical signal transferred through a coaxial cable has limitations like interference, cross talk, less security which can be overcome by transmitting the data using a light signal through an optical fiber [8]. Different bandwidth is preferred for the transmission of data through an optical fiber and mmw has become preferably suitable wave range due to its high bandwidth and efficiency [9]. The optical fiber has better transmission ability of mmw signal due its robustness and simple configuration leads to a low cost experimental setup [10], [11]. In order to generate mmw signal in optical domain, external modulators are preferred due to its ability to multiply and transform the frequency from a lower radio frequency (RF) signal into a mmw signal [12], [13]. By giving the proper DC bias, the optical mmw carrier generated is four times the frequency of the local oscillator signal [14]. Recently phase modulation is used for the generation of $\mathrm{mmw}$ due to its stable operation and carrier suppression is performed by adjusting the phase of the carrier signal [15], [16]. The experiments done recently is based on frequency doubling by using single phase modulation scheme with data rate of 2 - Gbaud over $22 \mathrm{~km}$ [17] and the data rate of $110 \mathrm{Gbps}$ is transmitted for a distance of $80 \mathrm{~km}$ using optical fiber technology [18]. The recent methods used to generate mmw using phase modulation scheme requires high cost for implementation and demands use of external modulator to produce the desired mmw signal. In this paper an experimental setup is introduced by using PM technique to generate optical mmw based on frequency octupling with a $\mathrm{LiNbO}_{3}$ MZM. By adjusting the bias voltage, a data rate of $100 \mathrm{Gbps}$ through a single mode fiber is achieved through a distance of $70 \mathrm{~km}$ with better efficiency. 


\section{EXPERIMENTAL SETUP AND ANALYSIS}

The figure 1 shows the arrangement used to generate mmw based on frequency octupling in experiment basis. In this setup a continuous wave (CW) laser with $193.1 \mathrm{THz}$ frequency is given as input to the $\mathrm{LiNbO}_{3}$ MZM with a $10 \mathrm{GHz}$ frequency from a source generator and to the phase modulator.

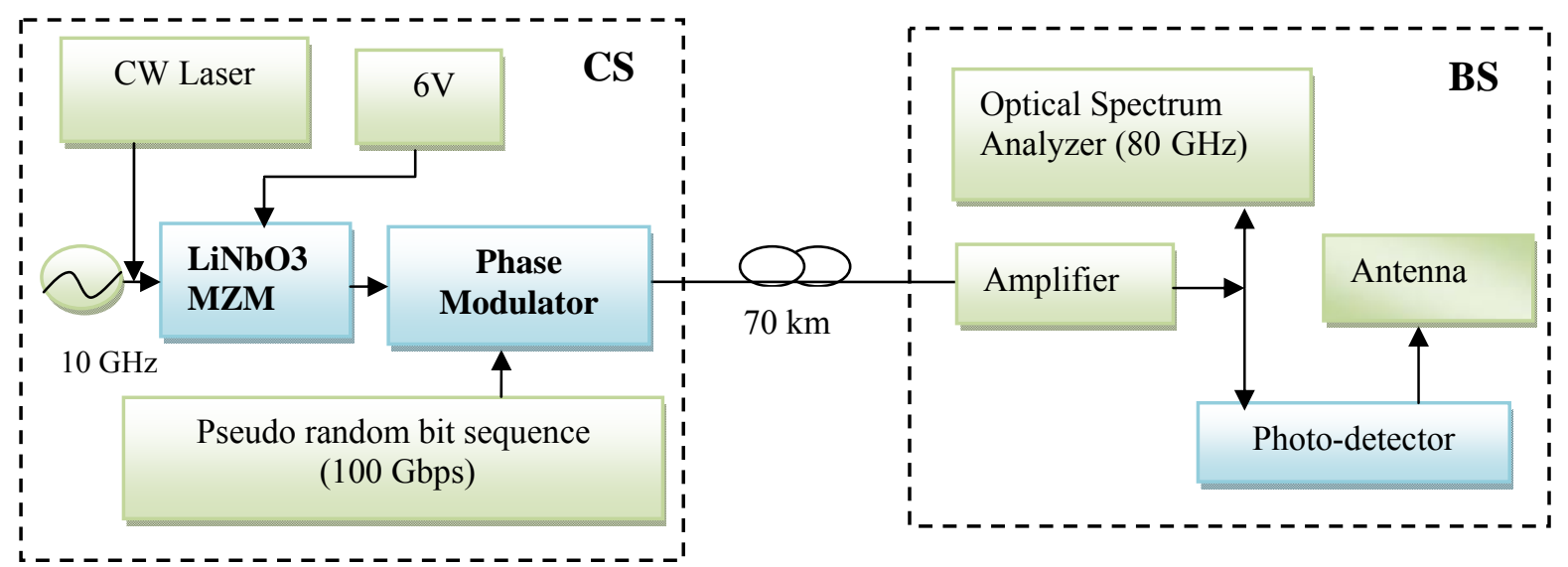

Fig. 1. Experimental setup for mmw generation based on frequency Octupling

In the modulator the bias and modulation voltage is set as $9 \mathrm{~V}$ and the phase deviation for the phase modulator is set as $90^{\circ}$. The data rate of $100 \mathrm{Gbps}$ is incorporated with the phase modulator and thus the output coming from the whole setup in the central station (CS) is a carrier suppressed mmw based on frequency octupling. The carrier signal and the even order optical sidebands are suppressed by the phase modulator so that the resultant signal has a frequency which is eight times that of the electrical signal.

The modulator electric field applied is expressed as

$$
E_{\text {in }}(t)=E_{0} \cos \left(\omega_{0} \mathrm{t}\right)
$$

The voltage of the millimeter wave signal is given as

$$
V(t)=V_{e} \cos \left(\omega_{c} t+\varphi_{1}\right)
$$

Where $E_{0}$ is the electric field amplitude while $V_{e}$ and $\varphi_{1}$ are amplitude and phase of electrical signal respectively. The bias voltage of phase modulator is varied in order to suppress all the even optical sidebands, the electric field output of the modulator can be expressed by

$$
\begin{aligned}
& \left.E_{\text {out } 1}(t)=E_{0} \cos \left(\omega_{o} t\right)\left(\pi / 2+\beta_{1} \cos \left(\omega_{c} t+\varphi_{1}\right)\right]\right) \\
& \approx B_{1}\left(\beta_{1}\right) E_{0} \cos \left(\left(\omega_{o}-\omega_{c}\right) t-\varphi_{1}\right)+\cos \left(\left(\omega_{o}+\omega_{c}\right) t+\varphi_{1}\right)
\end{aligned}
$$

Where $\beta_{1}=\left(V_{e} / V_{\pi 1}\right) \cdot \frac{\pi}{2}$, is the phase modulation index of the MZM.

$B_{1}$ is the first order Bessel function of the first kind, $\beta_{1}$ is the phase modulation index. $\varphi_{1}$ and $\varphi_{2}$ are the critical phase of the signal applied to the first and second modulator respectively.

The suppressed optical mmw signal is transmitted through a $70 \mathrm{~km}$ single mode fiber to reach the base station (BS). In the BS an amplifier is fixed as the first component so that the mmw coming from the optical fiber will fall directly on the amplifier in order to prevent the signal from fading. The optical signal from the amplifier is given as the input to the photo-diode to convert it into an electrical signal so that the signal can be given to the antenna for further transmission. In between an amplifier and the photo-diode an optical spectrum analyzer is connected to check whether the generated signal is preserved through the transmission. The final output from the photodiode is expressed mathematically as

$$
V_{\text {out }}(t)=B_{1}^{2}\left(\beta_{1}\right) B_{1}^{2}\left(\left(\beta_{2}\right) E_{0}^{2} \cos \left(8 \omega_{c} t+4 \varphi_{1}+4 \varphi_{2}\right)\right.
$$

It is evident from the above expression that the proposed phase modulation scheme achieves frequency octupling. 


\section{EXPERIMENTAL RESULTS}

The laser frequency of $10 \mathrm{GHz}$ is used as the input with a $10 \mathrm{GHz}$ signal generator to the MZM modulator, in the modulator a $9 \mathrm{~V}$ is applied and a $90^{\circ}$ phase shift is given for the source signal in order to generate the mmw signal. The output of the modulator converts the $10 \mathrm{GHz}$ carrier signal into $80 \mathrm{GHz}$ with a data rate of $100 \mathrm{Gbps}$ and performs a error free transmission through a $70 \mathrm{~km}$ long single mode fiber. The conversion of frequency is clearly observed in the optical and RF spectrum analyzer. The quality as well as the efficiency of the signal is analyzed by using the visualizers and in the BER analyzer the value of the Q-factor is high which indicates that the energy loss is low during transmission.

The figure 2 shows the observational results of the generated optical signal in terms of optical spectrum, electrical spectrum, eye diagram and Q-factor.

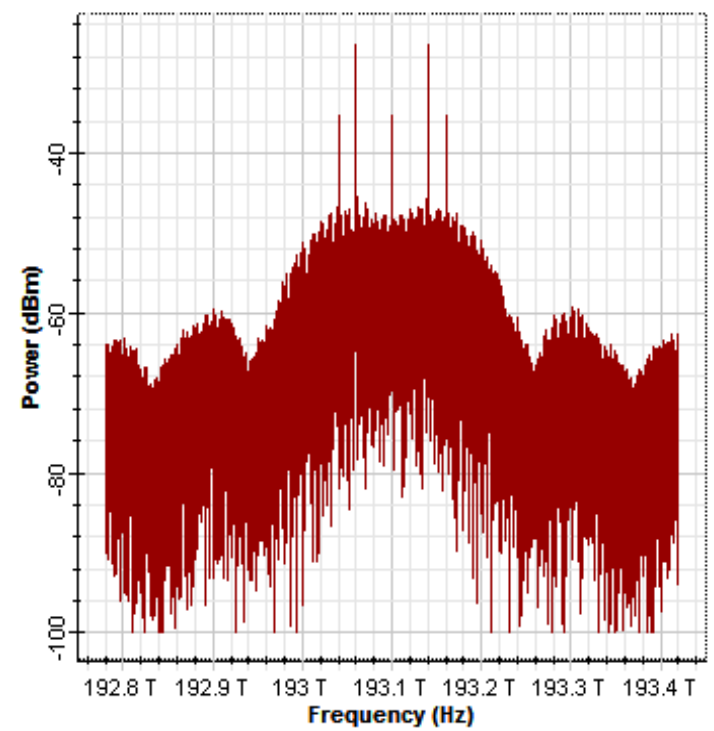

(a) Optical Spectrum

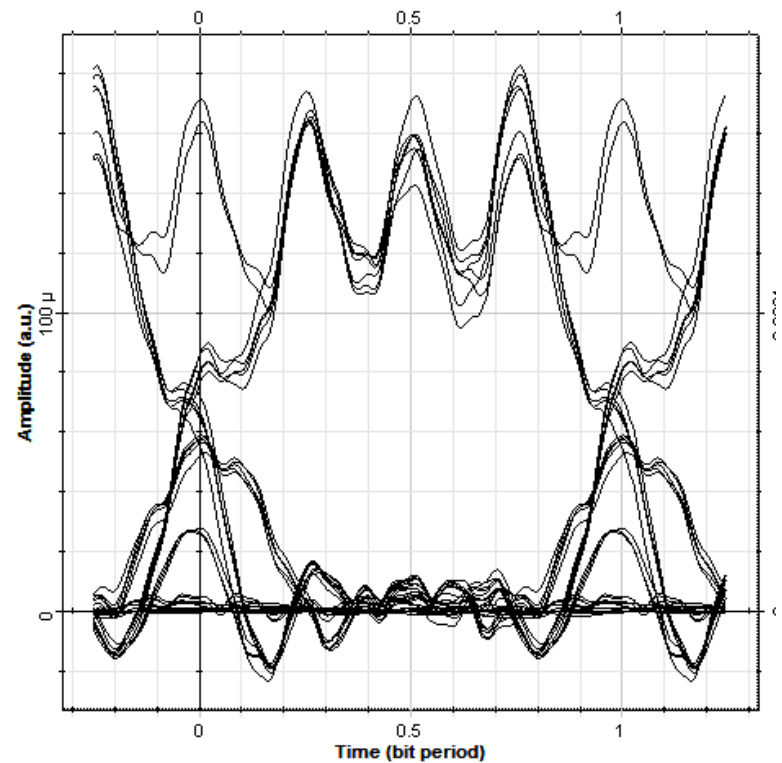

(c) Eye diagram

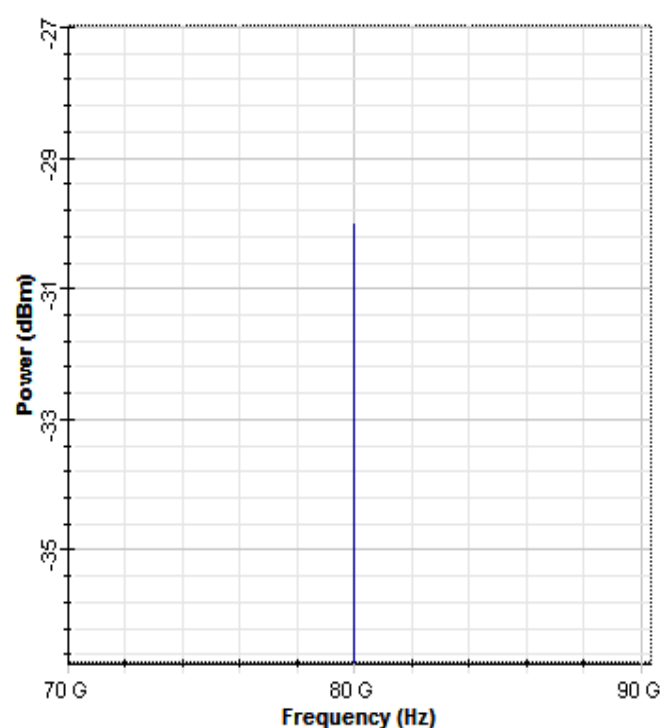

(b) Electrical Spectrum

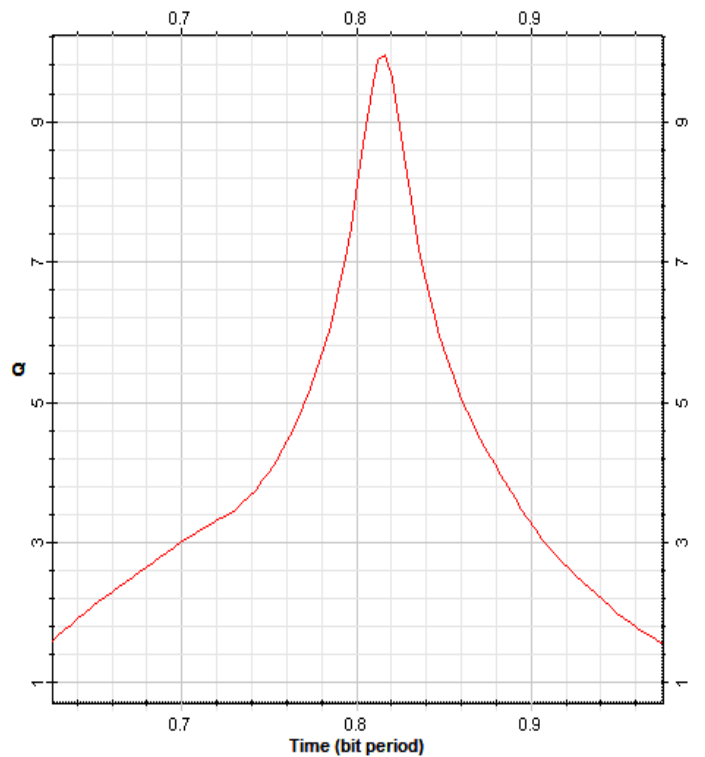

(b) Q- Factor

Fig. 2. Observed results after the transmission of optical mmw signal over $70 \mathrm{~km}$. 
The optical spectrum and the electrical spectrum of the generated mmw signal for the frequency $80 \mathrm{GHz}$ at a distance of $70 \mathrm{~km}$ is shown in figure $2(\mathrm{a}, \mathrm{b})$ and the eye diagram shown in fig. 2 (c) has a wide opened structure like an eye which clearly indicates the data transmitted using optical signal through an $60 \mathrm{~km}$ optical fiber is preserved and can be reconstructed effectively. The high value of Q-factor shown in figure 2 (d) obtained by using BER analyzer indicates the less energy loss occurred during the transmission of $\mathrm{mmw}$ from an optical fiber.

\section{FIBER LENGTH VERSUS QUALITY FACTOR}

The quality factor plays an important role in the transmission of signal through an optical fiber which graphically represents the energy loss during the transmission of $\mathrm{mmw}$ data signal. In table 1 the iteration method is used between the length of the fiber and the quality factor. By using this table it is clear that the energy loss occurs due to fading effect during the transmission of $100 \mathrm{Gbps}$ mmw signal for different fiber length. From table 1 analysis up to the length of $60 \mathrm{~km}$ the quality factor is maintained above the value range of $50 \mathrm{~km}$ but for $70 \mathrm{~km}$ a drastic change in the quality factor is observed.

TABLE. I. FIBER LENGTH VERSUS QUALITY FACTOR

\begin{tabular}{|c|c|}
\hline Fiber length in km & Quality Factor \\
\hline 30 & 55.331 \\
\hline 40 & 54.538 \\
\hline 50 & 54.432 \\
\hline 60 & 53.764 \\
\hline 70 & 49.212 \\
\hline
\end{tabular}

From table 1, it is understood that the drastic change in the value of quality factor from $60 \mathrm{~km}$ to $70 \mathrm{~km}$ is due to fading effect. To avoid this fading effect an amplifier is used in the base station and thus the range of quality factor is successfully maintained for a length of $70 \mathrm{~km}$ distance and it is shown in table 2 . The use of an amplifier in this setup will help to reduce the fading effect and the fiber length can be increased for better data transmission.

TABLE. II. FIBER LENGTH VERSUS QUALITY FACTOR USING AN AMPLIFIER

\begin{tabular}{|c|c|}
\hline Fiber length in $\mathbf{~ k m}$ & Quality Factor \\
\hline 50 & 54.432 \\
\hline 60 & 53.764 \\
\hline 70 (using an amplifier) & 54.212 \\
\hline
\end{tabular}

\section{BER VERSUS EXTINCTION RATIO}

The table 3 shows bit error rate (BER) versus extinction ratio done as an experiment basis to identify the changes occur in the data signal transmission to the base station. This experiment helps to describe the need of an extinction ratio to achieve error free transmission. The values for the extinction ratio is given in the modulator used to generate mmw and the BER is analyzed by using BER analyzer. According to the figure 3 analysis when extinction ratio increases the BER get reduced and this shows the prevention of message signal from losing throughout the distance of an optical fiber.

TABLE. III. BER VERSUS EXTINCTION RATIO

\begin{tabular}{|c|c|}
\hline Extinction Ratio & Bit Error Rate (BER) \\
\hline 10 & $10^{-15}$ \\
\hline 20 & $10^{-10}$ \\
\hline 30 & $10^{-19}$ \\
\hline 40 & $10^{-15}$ \\
\hline 50 & $10^{-9}$ \\
\hline 60 & $10^{-15}$ \\
\hline 70 & $10^{-21}$ \\
\hline 75 & $10^{-28}$ \\
\hline
\end{tabular}




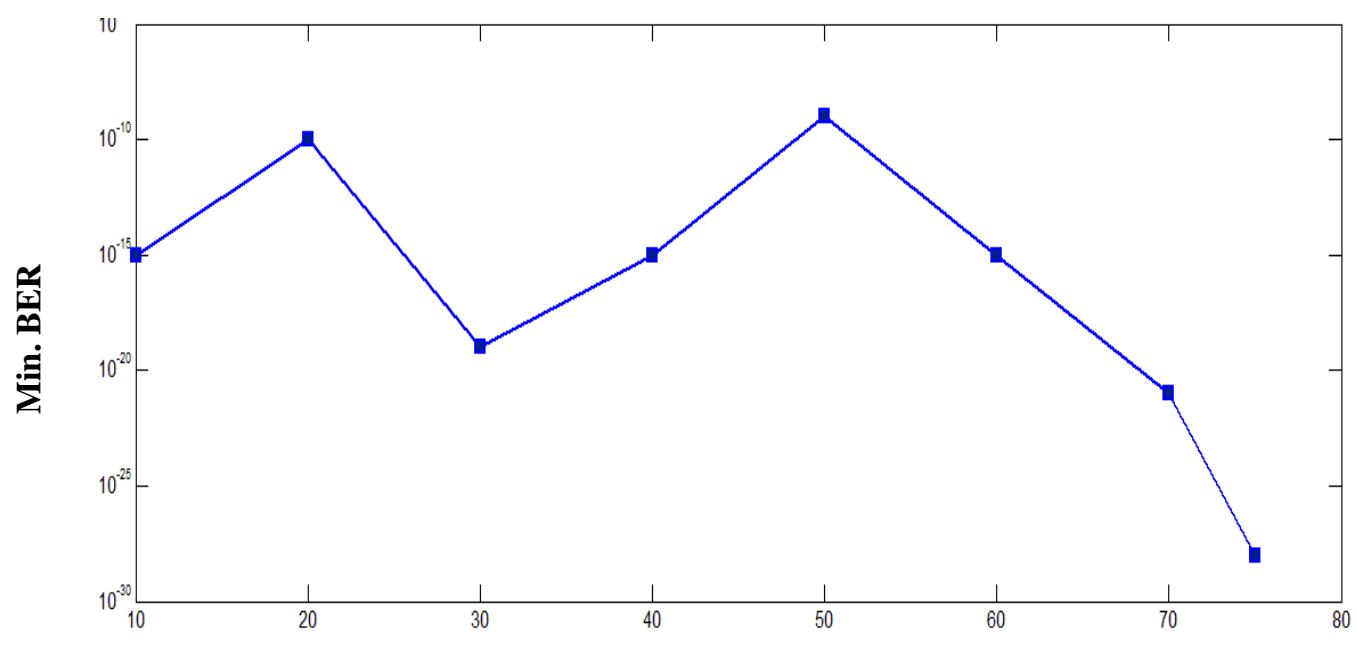

Extinction Ratio

Fig. 3. Graphical representation of Extinction ratio versus BER

\section{CONCLUSION}

A novel method for mmw generation using phase modulation with a single $\mathrm{LiNbO}_{3}$ intensity modulator based on frequency octupling is proposed. A data rate of $100 \mathrm{Gbps}$ is successfully transmitted through a single mode fiber for a length of $70 \mathrm{~km}$ and this cost effective model is analyzed to ensure the robustness of the generated mmw signal. The proposed work has a simple setup which is efficient and provides error free transmission of mmw through an optical fiber. The quality of the generated mmw signal for a distance of $70 \mathrm{~km}$ is verified by using the spectrum analyzers and the simulation result ensures the quality of the generated $\mathrm{mmw}$ signal. The results on Fiber length versus Quality factor and BER versus Extinction ratio gives more detailed information about the robustness of the proposed method and thus achieves a good quality mmw signal in the receiver side.

\section{REFERENCES}

[1] S. Chen. \& J, Zhao., The requirements, challenges and technologies for $5 \mathrm{~g}$ of terrestrial mobile telecommunication, IEEE Communication Magazine, 52(5), pp. 36-43, May 2014.

[2] X, Li., J, Xiao. \& J. Yu., Long-distance wireless mm-wave signal delivery at W-band, Journal of Lightwave Technology, 34(1), pp. 1 $-8,2016$.

[3] A, Chowdhury., H,Chien., Y,Hsueh. \& G.K, Chang., Advancedsystem technologies and field demonstration for in-building optical wireless network with integrated broadband services, Journal of Lightwave Technology, 27(22), pp. 1920-1927, 2009.

[4] X, Li., J, Yu. \& J, Xiao., Demonstration of ultra-capacity wireless signal delivery at W-band, Journal of Lightwave Technology, 4(1), pp. 180-187, Jan. 2016.

[5] G. K, Chang., A, Chowdhury., Z, Jia., H. C, Chien., M. F. Huang., J,Yu. \& G, Ellinas., Key technologies of WDM-PON for future converged optical broadband access networks, Journal of Optical Communication Networks, 1(4), pp. C35-C50, 2009.

[6] A, Khawaja. \& M. J. Cryan., Characterization of multimode fiber for use in millimeter wave radio-over-fiber systems, Microwave Optical Technology Letters, 50, pp. 2005-2007, 2008.

[7] Junwen Zhang., Jianjun Yu., Nan Chi., Ze Dong., Xinying Li. \& Gee-Kung Chang., Multichannel 120-Gb/s data transmission over 2 X 2 MIMO fiber-wireless link at W-band, IEEE Photonics Technology Letters, 25(8), pp. 780-783, 2013.

[8] J, J, V, Olmos., T, Kuri. \& K. Kitayama., Dynamic Reconfigurable WDM 60-GHz Millimeter-Waveband Radio-Over-Fiber Access Network: Architectural Considerations and Experiment, Journal of Lightwave Technology, 25, pp. 3374, 2007.

[9] J. Wells., Faster than fiber, The future of multi-Gb/s wireless, IEEE Microwave Magazine, 10, pp.104-112, 2009.

[10] Ma, Jianxin., Zhan, Yu. \& Yu Chongxiu., $64 \mathrm{Ghz}$ optical millimeter wave generation by octupling 8 Ghz local oscillator via a nested $\mathrm{LiNbO}_{3}$ modulator, Optics and Laser Technology, 42, pp. 264-268, 2010.

[11] L, Smoczynski. \& M, Marciniak., A comparison of different radio over fiber system concept with regard to application in mobile internet and multimedia, Proceedings of $4^{\text {th }}$ International Conference for Transparent Optical Networks, 1, pp. 211-213, 2002.

[12] J, Yu., Z, Jia., T, Wang. \& G, K, Chang., Centralized lightwave radio-over-fiber system with photonic frequency quadrupling for highfrequency millimeter-wave generation, IEEE Photonics Technology Letters, 19(19), pp.1499-1501, Oct. 2007.

[13] M, Weib., M, Huchard., A, Stohr., B, Charbonnier., S, Fedderwitz. \& D.S, Jager., $60 \mathrm{GHz}$ photonic millimeter-wave link for short- to medium range wireless transmission up to $12.5 \mathrm{~Gb} / \mathrm{s}$, Journal of Lightwave Technology, 26(15), pp. 2424-2429, 2008.

[14] K, Kitayama., A, Maruta. \& Y, Yoshida., Digital coherent technology for optical fiber and radio-over-fiber transmission systems, Journal of Lightwave Technology, 32(20), pp. 3411 -3420, Oct. 2014.

[15] C, Qingjiang., Simultaneous generation and transmission of downstream multiband signals and upstream data in a bidirectional radio over-fiber system, IEEE Photonics Technology Letters, 20(3), pp.181-183, Feb. 2008.

[16] Y, Hsueh., Z, Jia., H, Chien., J, Yu. \& G. K, Chang., A novel bidirectional 60-GHz radio-over-fiber scheme with multiband signal generation using a single intensity modulator, IEEE Photonics Technology Letters, 21(18), pp. 1338-1340, Sep. 2009. 
[17] Lun Zhao., Jianguo Yu., Lei Chen., Ping Min., Jia Heng Li. \& Renfan Wang., 16QAM Vector Millimeter-Wave signal Generation Based on Phase Modulator With Photonic Frequency doubling and Precoding, IEEE Photonic Journal, 8(2), April 2016.

[18] Xinying Li. \& Jianjun Yu., Fiber wireless transmission system of 108-Gb/s data over 80-km fiber and2 X 2 MIMO wireless links at100 GHz W-Band frequency, Optics Letters, 37(24), pp.5106-5108, Dec. 2012.

\section{AUTHOR PROFILE}

Anand Prem P K, is a full time Research Scholar at Anna University, Chennai, Tamil Nadu, India. He is doing research in the area of Optical Millimeter Wave Generation under the guidance of Dr. Arvind Chakrapani.

Dr. Arvind Chakrapani, is working as Associate Professor of Electronics and Communication Department, Karpagam College of Engineering, Coimbatore, Tamil Nadu, India. 